\title{
Les traitements thermiques appliqués aux produits laitiers et leurs conséquences dans le domaine azoté
}

(fin)

par

Jean ADRIAN

Centre de Recherches sur la Nutrition, C.N.R.S. - 92190 Bellevue

\section{L'EVOLUTION DE LA QUALITE PROTIDIQUE DANS LES POUDRES DE LAIT*}

On peut considérer les poudres de lait comme les produits industriels les plus sensibles à la réaction de Maillard. Cette situation résulte de divers facteurs fondamentaux et technologiques :

- richesse en lactose et réactivité des protéines du sérum,

- modalités de l'opération : pré-chauffage et technique d'évaporation de l'eau : vaporisation d'un brouillard de lait dans une tour d'air chaud (procédé Spray) ou passage d'une pellicule de lait sur un cylindre chauffé à la vapeur (procédé Hatmaker),

- caractère hygroscopique du lactose qui permet une réhydratation partielle de la poudre de lait et facilite, par là-même, une réaction sucre - acides aminés.

La réaction de Maillard risque de se produire aussi bien lors de la fabrication que pendant la conservation de la poudre. Ce phénomène peut également se développer avec intensité lorsque la poudre est soumise à des traitements thermiques variés. Nous examinerons donc les trois étapes au cours desquelles la qualité protidique du lait peut être endommagée.

\section{Evolution protidique du lait au moment du séchage}

La réaction de Maillard étant autocatalytique (Adrian, 1973 b), l'intensité d'un premier chauffage détermine l'ampleur des répercussions des traitements ultérieurs. C'est pourquoi, les conséquences du séchage dépendent partiellement de la nature du pré-chauffage subi par le lait (Henry et Kon, 1947 ; Shillam et Roy, 1963 ; Van den Bruel et al., 1972).

\footnotetext{
* Dans cet exposé, le terme de poudre de lait s'applique au produit élaboré à partir du lait écrémé, sauf mention particulière.
} 
Dans les cas les plus habituels, le pré-chauffage du lait n'entraîne pas de répercussions significatives sur la qualité de la poudre de lait, bien que la lysine disponible tende à diminuer lorsque le pré-traitement devient sévère.

En réalité, lorsqu'on procède à un traitement thermique peu préjudiciable, pour mettre en relief ses effets éventuellement défavorables on a intérêt à recourir à des organismes connus pour l'extrême sensibilité de leur appareil digestif. A ce sujet, le veau pris à la naissance se comporte comme un "révélateur » de choix permettant de déceler des dommages très légers provoqués par un chauffage. L'exemple ci-dessous en fournit une remarquable illustration, qui ne pourrait être retrouvée avec les autres animaux de laboratoire :

\begin{tabular}{ll|c|c}
\hline & \multicolumn{2}{|c}{ Poudre de lait Spray pré-chauffée à } \\
\cline { 3 - 4 } & & $77^{\circ}$ C pendant $15 \mathrm{~s}$ & $74^{\circ} \mathrm{C}$ pendant $30 \mathrm{mn}$ \\
\cline { 3 - 4 } & & \\
\cline { 3 - 3 } & & 89,0 & 80,5 \\
Digestibilité globale (p. 100) & 77,5 & 60,2 \\
Digestibilité azotée & (p. 100) & 66 & 66 \\
Valeur biologique & & & \\
\hline
\end{tabular}

D'une manière générale, le procédé Spray, par atomisation, respecte presque intégralement la lysine du lait tandis que la technique sur cylindre l'endommage dans des proportions variables (Pion et Rerat, 1962 ; Mac Donald, 1966 ; Holm, 1971 ; Van den Bruel et al., 1972 ; etc. $)^{*}$.

Ainsi, au cours d'une enquête portant sur divers échantillons commerciaux, Erbersdobler et Zucker (1966) constatent qu'en moyenne les poudres atomisées renferment 10 p. 100 de plus de lysine disponible que celles séchées sur cylindres; une différence de 20 p. 100 n'est pas exceptionnelle.

\footnotetext{
* Diverses observations de Fink $(1958,1959$ et 1963) se rapportent au développement d'une nécrose hépatique chez des animaux recevant $10 \mathrm{p} .100$ de poudre de lait, la mortalité étant proportionnelle à la sévérité du mode de séchage. Elle est inférieure à 1 p. 100 chez les sujets consommant du lait frais, de 40 p. 100 chez ceux qui reçoivent une poudre Spray et atteint 70 p. 100 avec la poudre séchée sur cylindre.

En réalité, cet état pathologique ne reflète pas l'existence d'une substance toxique mais une indisponibilité du sélénium dans les poudres de lait : la nécrose est totalement prévenue par une administration quotidienne de $0,4 \mu \mathrm{g}$ de sélénite de sodium. Une situation analogue - et relevant probablement d'un mécanisme identique - s'observe avec la levure torula (in Champagnat et Adrian, 1974).
} 
Les préjudices enregistrés avec les sécheurs à cylindres dépendent du type d'appareil utilisé et de son réglage. Selon Pion et Rerat (1962), le sécheur à gicleur qui projette le lait sur le cylindre produit une poudre soutenant la comparaison avec les produits Spray tandis que les cylindres " à bourbier " dans lesquels le tambour plonge dans le liquide fournissent une poudre accusant une destruction de 18 p. 100 de la lysine.

Par ailleurs, les modalités d'emploi des cylindres exercent une influence considérable signalée de divers côtés (Mauron et al., 1955 ; Holm, 1971 ; Van den Bruel et al., 1972 ; Erbersdobler et Duemmer, 1971). Ces derniers illustrent ainsi les effets du réglage du tambour sur la qualité azotée de la poudre :

\begin{tabular}{|c|c|c|c|c|}
\hline \multicolumn{2}{|c|}{ Modalités du séchage } & \multirow{2}{*}{\multicolumn{2}{|c|}{$\begin{array}{c}\text { Lysine } \\
\text { (p. } 100 \text { des protéines) }\end{array}$}} & \multirow{3}{*}{$\begin{array}{c}\text { Lysine } \\
\text { disponible } \\
\text { (p. 100) }\end{array}$} \\
\hline \multirow{2}{*}{$\begin{array}{l}\text { Température } \\
\text { du tambour }\end{array}$} & \multirow{2}{*}{$\begin{array}{l}\text { Temps de contact } \\
\text { de la pellicule de lait } \\
\text { (en s) }\end{array}$} & & & \\
\hline & & totale & disponible & \\
\hline $116^{\circ} \mathrm{C}$ & 4 & 8,9 & 6,6 & 74 \\
\hline $120^{\circ} \mathrm{C}$ & 7,5 & 5,8 & 2,2 & 38 \\
\hline
\end{tabular}

En bref, le séchage par atomisation apparaît une technique reproductible et sans conséquences fâcheuses notables sur le plan protidique tandis que l'emploi de cylindres fait figure d'une technique plus aléatoire quant à ses résultats et s'accompagnant presque inévitablement d'un préjudice en ce qui concerne la disponibilité de la lysine.

Dans la pratique, Prahl et Tauefel (1967) retiennent trois catégories de poudres de lait, classées en fonction de leurs caractéristiques physico-chimiques :

\begin{tabular}{l|c|c|c}
\hline \multicolumn{1}{c}{$\begin{array}{c}\text { Qualificatif } \\
\text { de la } \\
\text { poudre }\end{array}$} & $\begin{array}{c}\text { Digestibilité } \\
\text { azotée } \text { in vitro } \\
(\mathrm{p} .100)^{*}\end{array}$ & $\begin{array}{c}\text { Teneur } \\
\text { en lysine } \\
\text { (p. 100 du lait })\end{array}$ & Brunissement** \\
\cline { 2 - 3 } & supér. à 87 & supér. à 90 & $70-85$ \\
Non endommagée & $70-87$ & $60-90$ & $30-70$ \\
Légèrement endommagée & infér. à 60 & infér. à 30 \\
Fortement endommagée & infér. à 70 & \\
\hline * Digestion à l'aide de pepsine et pancréatine. \\
** p. 100 de lumière réfléchie, mesuré avec un Leukometer Zeiss. \\
\hline
\end{tabular}


Mais le test probablement le plus immédiat pour classer la qualité d'une poudre de lait demeure la mesure de sa solubilité, celle-ci diminuant parallèlement avec la détérioration de sa qualité protidique (Richards, 1963 ; Erbersdobler et Zucker, 1966 ; Prahl et Tauefel, 1967 ; etc.). Ce genre d'évolution est reportée dans la figure 2 donnant à la fois le taux d'insolubilisation du lait (volume de sédiment) et celui d'azote aminé libre, au cours d'une conservation à $37^{\circ} \mathrm{C}$ et sous une humidité relative de 55 p. 100 .

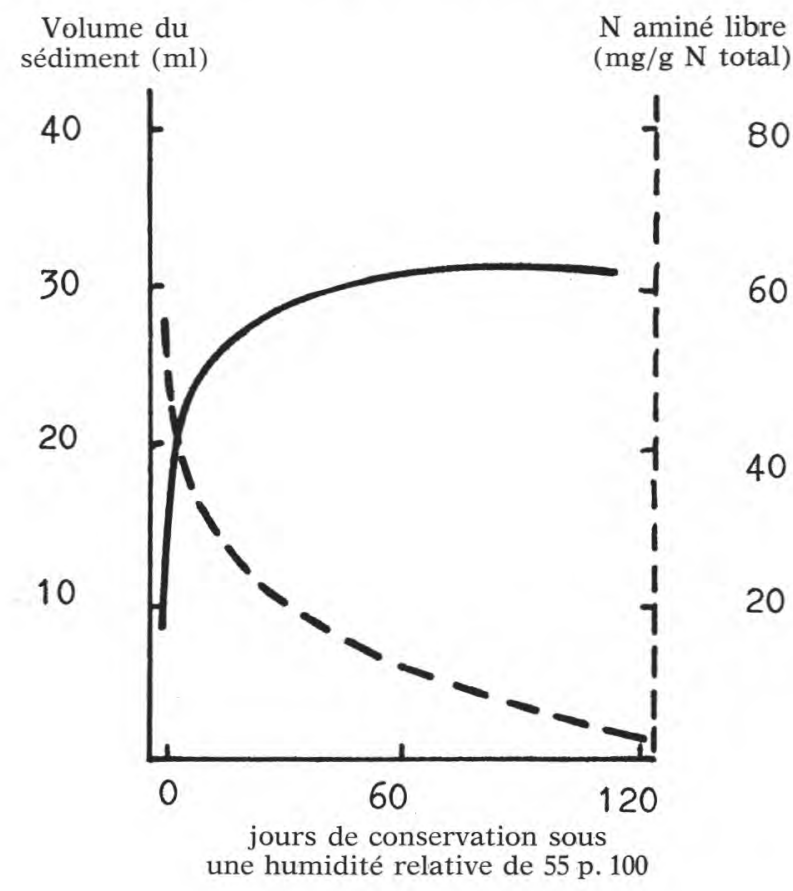

fig. 2

Evolution de la solubilité d'une poudre de lait et de son taux d'azote aminé libre, au cours d'une conservation à $37^{\circ} \mathrm{C}$ (d'après Richards, 1963).

\section{Evolution protidique de la poudre de lait pendant sa conservation}

Les poudres de lait sont sujettes à de graves altérations lors des périodes de conservation, le fait ayant été mis en lumière depuis longtemps par l'étude remarquable de Henry, Kon, Lea et White (1946, 1948).

Le principal agent responsable de la dégradation protidique des poudres de lait est leur caractère hygroscopique qui leur permet d'atteindre rapidement un degré d'humidité très préjudiciable pour leur stabilité : sous une humidité relative de 80 p. 100, la teneur en eau peut passer de 4,7 à 6,2 p. 100 en 12 h (Henry et al., 1948) et 
atteindre un plafond de l'ordre de $12-14$ p. 100 en 2 ou 3 semaines (Nakanishi et Itoh, 1966).

Cette hydratation - facteur indispensable à la réaction de Maillard (Loncin et al., 1965) — va grandement accélérer les réactions chimiques au sein de la poudre de lait en facilitant la mobilité de ses constituants. Les valeurs suivantes montrent le rôle respectif de l'eau et de la température sur l'intensité des réactions chimiques d'une poudre de lait (Henry et al., 1948) :

Evolution de la poudre de lait au cours de sa conservation (en mg pour $100 \mathrm{~g}$ de poudre et pour $100 \mathrm{j}$ )

\begin{tabular}{|c|c|c|c|c|c|c|}
\hline Température de stockage & \multicolumn{3}{|c|}{$37^{\circ} \mathrm{C}$} & \multicolumn{3}{|c|}{$20^{\circ} \mathrm{C}$} \\
\hline Humidité de la poudre (p. 100) & 7,3 & 4,7 & 2,9 & 7,3 & 4,7 & 2,9 \\
\hline $\mathrm{O}_{2}$ absorbé & 245,0 & 3,25 & 2,5 & 12,5 & 0,95 & 0,7 \\
\hline emballage normal & 98,0 & 0,85 & 0,4 & 6,0 & 0,2 & 0,1 \\
\hline sous azote & 10,0 & 0,45 & 0,2 & - & $<0,1$ & $<0,1$ \\
\hline
\end{tabular}

Le gaz carbonique dégagé est - au moins partiellement - une manifestation spécifique de la réaction de Maillard, plus précisément de la dégradation de Strecker. En effet, il existe un parallélisme étroit entre le degré d'humidité de la poudre et l'intensité de la réaction de Maillard qui s'y développe : d'une manière schématique, la réaction demeure nulle pour des teneurs en eau inférieures à 3 p. 100 , elle est très faible dans l'intervalle compris entre 3 et 5 p. 100 et atteint son intensité maximale pour des humidités comprises entre 7,5 et 12 p. 100 environ (Henry et al., 1948 ; Kisza et al., 1966 ; Erbersdobler et Zucker, 1966 ; Erbersdobler, 1970 ; etc.). Ce dernier a établi la relation suivante entre l'humidité et l'évolution de la lysine d'une poudre de lait, après 10 semaines à $30^{\circ} \mathrm{C}$ :

\begin{tabular}{|c|c|c|c|c|}
\hline Humidité de la poudre (p. 100) & 4 & 6 & 8 & 10 \\
\hline $\begin{array}{l}\text { En p. } 100 \text { des protéines: } \\
\text { lysine utilisable }\end{array}$ & $\begin{array}{c}7,0 \\
(100)\end{array}$ & $\begin{array}{c}6,5 \\
(93)\end{array}$ & $\begin{array}{c}4,8 \\
(69)\end{array}$ & $\begin{array}{c}5,5 \\
(79)\end{array}$ \\
\hline lysine combinée & $\begin{array}{c}0,5 \\
(100)\end{array}$ & $\begin{array}{c}0,8 \\
(160)\end{array}$ & $\begin{array}{l}2,6 \\
(520)\end{array}$ & $\begin{array}{c}2,0 \\
(400)\end{array}$ \\
\hline
\end{tabular}


Henry et al. (1948) donnent un tableau très complet des manifestations de la réaction de Maillard développée dans des conditions de stockage défavorables (humidité de la poudre Spray $=7,3 \mathrm{p} .100$; $60 \mathrm{j}$ à $\left.37^{\circ} \mathrm{C}\right)(\mathrm{tab} .3)$ :

\section{TABLEAU 3}

Evolution d'une poudre de lait Spray au cours d'une conservation de $60 \mathrm{j}$ à $37^{\circ} \mathrm{C}$, avec une humidité de $7,3 \mathrm{p} .100^{*}$

Humidité (p. 100)

Equilibre d'humidité relative (p. 100)

Solubilité à $20^{\circ} \mathrm{C}$ (p. 100)

pH

Protéines de la fraction soluble de la poudre, en p. 100 de l'azote total de la poudre fraîche :

$\mathrm{N}$ caséine

$\mathrm{N}$ albumine

$\mathrm{N}$ globuline

N protéose-peptone

$\mathrm{N}$ non protidique

Pouvoir réducteur (ferricyanure)

Sucre réducteur combiné aux protéines (en $\mathrm{mg}$ de lactose/g de mat. sèche)

$\mathrm{N}$ aminé libre (valeur relative)

Digestibilité azotée (p. 100)

C.E.P.

C.E.P. après addition de lysine

Valeur biologique

Valeur biologique après addition de lysine Flaveur

\begin{tabular}{|c|c} 
Poudre fraîche & Poudre conservée \\
\cline { 2 - 2 } 7,3 & \\
42 & 6,7 \\
99 & 70 \\
6,73 & 6,50 \\
& \\
& \\
80,7 & 6,8 \\
2,3 & 0 \\
3,7 & 4,6 \\
6,8 & 12,8 \\
6,5 & 6,7 \\
0,9 & 16,0 \\
6 & 49 \\
100 & 36 \\
91,2 & 86,0 \\
2,81 & 1,82 \\
2,59 & 2,55 \\
84,5 & 67,5 \\
76,4 & 80,1 \\
agréable & goût de colle \\
& désagréable et \\
& caramélisé \\
& \\
&
\end{tabular}

* d'après Henry et al. (1946).

- on enregistre un certain nombre de modifications physiques : brunissement, acidification et insolubilisation de la poudre de lait et de sa fraction caséine ;

- les modifications chimiques traduisent une réaction importante entre le lactose et les acides aminés, allant jusqu'au stade de la dégradation de Strecker, avec un dégagement de $\mathrm{CO}_{2}$ (conséquence de la décarboxylation des acides aminés); la valeur protidique est nettement amoindrie, aussi bien sa digestibilité azotée que son efficacité métabolique (V.B. ou C.E.P.).

De plus, le blocage de la lysine fait de cet acide aminé le facteur limitant de la poudre de lait conservée, comme le confirme la restauration de la valeur protidique par une supplémentation en lysine. 
En pratique, même dans des régions non réputées par la chaleur de leur climat, des altérations très sensibles sont enregistrées pendant les périodes de stockage des poudres de lait. Ainsi, en Angleterre, après 3 ans, la valeur biologique passe de 88,5 à 71,1 (Henry et al., 1946 b). En Allemagne, une poudre de lait conservée pendant 7 mois dans un sous-sol accuse une perte importante de sa qualité, la valeur biologique tombant de 83 à 51 (Schiller, 1956).

Même dans une chambre froide et à l'abri de l'humidité, une poudre de lait n'est pas parfaitement stabilisée comme le montrent les analyses de Bock et Wuensche (1965) dont les résultats sont condensés dans le tableau 4.

\section{TABLEAU 4}

Evolution d'une poudre de lait au cours d'une conservation en chambre froide à l'abri de l'humidité*

\begin{tabular}{|c|c|c|c|c|c|c|}
\hline & \multicolumn{3}{|c|}{$\begin{array}{c}\text { Poudre de lait } \\
\text { Spray }\end{array}$} & \multicolumn{3}{|c|}{$\begin{array}{l}\text { Poudre de lait séchée } \\
\text { sur cylindre }\end{array}$} \\
\hline & $\begin{array}{l}\text { fraî- } \\
\text { che }\end{array}$ & $\begin{array}{c}\text { après } \\
6 \\
\text { mois }\end{array}$ & $\begin{array}{c}\text { après } \\
12 \\
\text { mois }\end{array}$ & $\begin{array}{l}\text { fraî- } \\
\text { che }\end{array}$ & $\begin{array}{c}\text { après } \\
6 \\
\text { mois }\end{array}$ & $\begin{array}{c}\text { après } \\
12 \\
\text { mois }\end{array}$ \\
\hline Valeur biologique & 81,1 & 78,9 & 75,0 & 81,4 & 79,0 & 76,9 \\
\hline Lysine totale (p. 100 des protéines) & 9,0 & 9,0 & 6,8 & $(8,8)$ & 8,8 & 7,3 \\
\hline Lysine digestible (p. 100 des protéines) & 6,0 & 5,3 & 5,0 & 5,1 & 5,3 & 4,7 \\
\hline
\end{tabular}

* d'après Bock et Wuensche (1965).

Au total, même dans des climats tempérés les poudres de lait se révèlent endommagées par une conservation de quelques mois ou de l'ordre d'une année. Le préjudice s'amplifiera en fonction de la chaleur et de l'humidité. On peut alors imaginer facilement les altérations subies par les poudres de lait expédiées dans des régions chaudes, ainsi que cela a été fait à une époque en vue de distributions aux enfants : il n'est pas exagéré d'affirmer que ces envois avaient perdu une part appréciable de leur potentiel nutritionnel avant d'arriver au bénéficiaire.

A côté de cette évolution biochimique, on doit noter une modification physique dans les poudres de lait affectant sa fraction protidique et se traduisant par une insolubilisation massive de la caséine, notée dès 1948 par Henry et al. Ce phénomène a été analysé plus récemment dans une poudre de lait conservée sous une humidité relative de 80 p. 100 . On observe d'abord une insolubilisation plus rapide et plus importante de la caséine $\alpha_{s}$ que de la 
fraction $\beta$. Surtout, l'insolubilisation se fait sous forme de caséinate de calcium dont la composition est très proche de celle de la micelle native ; il en résulte que la fraction demeurant en solution est fortement déminéralisée comme le montrent les analyses de Nakanaishi et Itoh (1966):

Micelle de la caséine du lait

Après $20 \mathrm{j}$ de conservation : fraction protidique insoluble caséine encore soluble

Après $50 \mathrm{j}$ de conservation : fraction protidique insoluble caséine encore soluble

\begin{tabular}{|c|c}
$\begin{array}{c}\text { Calcium } \\
\text { (p. 100) }\end{array}$ & $\begin{array}{c}\text { Phosphore } \\
\text { (p. 100) }\end{array}$ \\
\hline 2,9 & 2,2 \\
& \\
2,99 & 1,72 \\
0,0 & 0,70 \\
& \\
3,08 & 1,74 \\
0,0 & 0,64 \\
\end{tabular}

On peut s'attendre à ce que cette insolubilisation progressive de la caséine en rende l'utilisation digestive plus lente sinon incomplète. Cette dénaturation physique pourrait contribuer, au moins partiellement, à la diminution de la digestibilité azotée maintes fois enregistrée dans les poudres de lait ayant subi une conservation.

En définitive, la valeur exceptionnelle du lait et la fragilité de sa poudre justifieraient donc amplement l'élaboration d'une politique de conservation lorsqu'un stockage de longue durée est prévu.

En premier lieu, on devrait introduire dans les emballages des capsules de substances desséchantes, de manière à maintenir l'humidité à un niveau ne dépassant pas 3 p. 100 environ ; deuxièmement, l'emballage devrait être opaque à la lumière et à l'oxygène ; troisièmement, le produit devrait être conservé sous gaz inerte ou sous vide ; quatrièmement, la poudre de lait devrait être maintenue à une température inférieure à $20^{\circ} \mathrm{C}$, dans la mesure du possible.

\section{Evolution protidique de la poudre de lait au cours des chauffages}

$\mathrm{Si}$ cette question semble revêtir un caractère quelque peu expérimental, elle n'est cependant pas totalement dénuée d'incidences pratiques. En effet, les poudres de lait sont appelées à subir directement ou non - des traitements thermiques dans des conditions variées : en boulangerie et en biscuiterie, dans les industries de produits infantiles, dans les industries d'alimentation animale, etc.

A priori, l'ensemble des phénomènes enregistrés lors du stockage de la poudre va se retrouver - avec une intensité accrue - dans le cas d'un traitement thermique. C'est ainsi que l'on va assister à une 
TABLEAU 5

Evolution de la valeur nutritionnelle d'une poudre de lait soumise à un grillage à $145^{\circ} \mathrm{C}^{*}$

\begin{tabular}{|c|c|c|c|c|c|c|c|}
\hline & \multirow{2}{*}{\multicolumn{2}{|c|}{$\begin{array}{l}\text { Poudre de lait } \\
\text { témoin }\end{array}$}} & \multicolumn{5}{|c|}{ Poudre de lait grillée à $145^{\circ} \mathrm{C}$ pendant } \\
\hline & & & \multicolumn{3}{|c|}{$25 \mathrm{mn}$} & \multicolumn{2}{|c|}{$50 \mathrm{mn}$} \\
\hline & seule & + méthionine & seule & + méthionine & + lysine & seule & + lysine \\
\hline Lysine totale $(\mathrm{mg} / \mathrm{g})$ & $\begin{array}{c}31,9 \\
(100)\end{array}$ & & $\begin{array}{c}7,5 \\
(23)\end{array}$ & & & $\begin{array}{c}4,6 \\
(14)\end{array}$ & \\
\hline $\begin{array}{l}\text { Lysine digestible libre** } \\
(\mathrm{mg} / \mathrm{g})\end{array}$ & $\begin{array}{l}20,9 \\
(100)\end{array}$ & & $\begin{array}{l}5,35 \\
(26)\end{array}$ & & & $\begin{array}{c}2,3 \\
(11)\end{array}$ & \\
\hline Méthionine totale $(\mathrm{mg} / \mathrm{g})$ & $\begin{array}{r}9,55 \\
(100)\end{array}$ & & $\begin{array}{l}9,60 \\
(101)\end{array}$ & & & $\begin{array}{l}9,50 \\
(99)\end{array}$ & \\
\hline $\begin{array}{l}\text { Méthionine digestible } \\
\text { libre }^{* *}(\mathrm{mg} / \mathrm{g})\end{array}$ & $\begin{array}{c}5,5 \\
(100)\end{array}$ & & $\begin{array}{l}4,25 \\
(77)\end{array}$ & & & $\begin{array}{l}3,15 \\
(57)\end{array}$ & \\
\hline C.E.P. & $\begin{array}{c}3,7 \\
(100)\end{array}$ & $\begin{array}{l}4,25 \\
(115)\end{array}$ & $\begin{array}{l}0,55 \\
(15)\end{array}$ & $\begin{array}{l}0,46 \\
(13)\end{array}$ & $\begin{array}{l}1,69 \\
(46)\end{array}$ & nég. & $\begin{array}{l}1,07 \\
(29)\end{array}$ \\
\hline
\end{tabular}

* d'après Adrian (1967), Frangne et Adrian (1967).

** acides aminés directement utilisables à la suite d'une attaque pepsine, trypsine et érepsine. 
destruction massive de lysine et à une baisse parallèle de la valeur nutritionnelle mesurée chez l'animal : la lysine devient le facteur limitant de la poudre de lait aussi bien après un autoclavage de $15 \mathrm{mn}$ à $120^{\circ} \mathrm{C}$ (Kraft et Morgan, 1952) qu'à la suite d'un chauffage de $25 \mathrm{mn}$ à $145^{\circ} \mathrm{C}$ (Adrian, 1967) (tab. 5).

D'après diverses observations (Ross et Krampitz, 1960 ; Schormueller et Andraess, 1962), le comportement de la lysine paraît dépendre au moins autant de la température de traitement que de la somme de chaleur appliquée : aux températures relativement basses le blocage serait le phénomène prédominant tandis que dans les traitements à plus haute température la destruction l'emporterait. Après $24 \mathrm{~h}$ à $105^{\circ} \mathrm{C}$ la perte de lysine totale est de $18 \mathrm{p} .100$ et celle de la lysine digestible de $42 \mathrm{p} .100$ après $102 \mathrm{~h}$ à $105^{\circ} \mathrm{C}$ les pertes respectives sont de 26 et 61 p. 100 .

$\mathrm{Au}$ cours d'un traitement thermique, le préjudice ne se limite pas à la lysine de la poudre de lait et aux acides aminés basiques : le tableau 5 fait ressortir le blocage d'une part appréciable de la méthionine ; de même, le tryptophane ne demeure pas insensible à l'autoclavage d'une poudre de lait (tab. 6) : lui aussi peut être partiellement bloqué au cours d'un chauffage ainsi que le démontre l'efficacité mesurée chez le rat ou la fraction hydrolysable in vitro par les systèmes protéolytiques du tube digestif (pepsine, trypsine et érepsine).

Cependant, ces phénomènes secondaires ne doivent pas faire perdre de vue la thermosensibilité sélective des acides aminés basiques (Ross et Krampitz, 1960) et les conséquences pratiques qui en résultent.

A titre d'illustration, on peut rappeler que la détérioration thermique des poudres de lait enlève tout intérêt protidique à la constitution de biscuits hyperazotés enrichis avec des poudres de lait. Au cours de la cuisson, la réaction de Maillard atteint une intensité telle que le biscuit otfre une qualité nutritionnelle bien inférieure à celle d'autres mélanges dont les composants sont plus stables thermiquement (Adrian et Frangne, 1969) :

Evolution de la qualité protidique de divers biscuits au cours de la cuisson (mélange cru $=100$ )

\begin{tabular}{l|c|c|c}
\hline $\begin{array}{c}\text { Addition } \\
\text { à la farine }\end{array}$ & C.E.P. & $\begin{array}{c}\text { Lysine } \\
\text { digestible }\end{array}$ & $\begin{array}{c}\text { Méthionine } \\
\text { digestible }\end{array}$ \\
\hline Farine d'arachide & 50 & 75 & 116 \\
Farine de poisson & 42 & 72 & 64 \\
Poudre de lait & 1 & 30 & 68 \\
\hline
\end{tabular}


TABLEAU 6. - Sensibilité du tryptophane dans la poudre de lait autoclavée*

\begin{tabular}{|c|c|c|c|c|c|}
\hline & \multirow{3}{*}{$\begin{array}{l}\text { Tryptophane } \\
\text { total }\end{array}$} & \multicolumn{4}{|c|}{ Tryptophane utilisable par } \\
\hline & & rat & \multicolumn{3}{|c|}{ L. arabinosus après hydrolyse avec } \\
\hline $\begin{array}{l}\text { Teneur de la poudre de lait témoin } \\
\text { (mg/g d'azote) }\end{array}$ & & & $\begin{array}{c}\text { pepsine } \\
\text { trypsine } \\
70\end{array}$ & $\begin{array}{c}\text { pepsine } \\
\text { trypsine } \\
\text { érepsine } \\
70\end{array}$ & pancréatine \\
\hline - poudre témoin & 100 & 100 & 100 & 100 & 100 \\
\hline - après $20 \mathrm{mn}$ d'autoclavage & 111 & 56 & 88 & 90 & 109 \\
\hline - après $40 \mathrm{mn}$ d'autoclavage & 107 & 59 & 77 & 81 & 114 \\
\hline
\end{tabular}

* d'après Pongpaew et Guggenheim (1968). 
Dans cet exemple, comme dans les valeurs du tableau 5 et du tableau 6 et de nombreux autres travaux, la chute de la qualité protidique mesurée sur l'animal est plus importante que la diminution du taux des acides aminés utilisables ou digestibles in vitro. Cette constatation offre une grande importance dans le domaine des répercussions physiologiques de la réaction de Maillard car elle démontre l'existence d'autres phénomènes que le simple blocage des acides aminés et leur destruction (Adrian, 1972).

\section{AUTRES MANIFESTATIONS DE LA REACTION DE MAILLARD DANS LES PRODUITS LAITIERS}

\section{Conséquences physico-chimiques}

La réaction de Maillard s'accompagne de phénomènes variés de nature physico-chimique (brunissement, fluorescence, acidification) qui se décèlent après le chauffage des laits* comme au cours des phases de conservation des laits liquides ou en poudre (Coulter et al., 1952).

a) Brunissement : Pour des raisons de qualité commerciale, le brunissement du lait est une manifestation qui a été très étudiée (Patton, 1955). Son incidence pratique est considérable puisqu'une coloration peut être détectée précocement, à la suite de traitements n'entraînant pratiquement pas de préjudices dans le domaine protidique (Ford et al., 1966) ; cependant, à ce point de vue, le lait U.H.T. constitue une exception puisqu'il ressort de la stérilisation plus blanc qu'avant l'opération (Burton, 1965).

Le brunissement se révèle directement proportionnel à certains facteurs :

- à l'intensité du traitement thermique ou à la durée de stockage du produit laitier (Tarassuk et Jack, 1948 ; Patton, 1952 ; Burton, 1965 ; etc.),

- à l'humidité de la poudre du lait (Tarassuk et Jack, 1948),

- au taux de lactose du lait, qui est l'élément indispensable au développement d'une coloration (Patton, 1952).

Ce phénomène est également favorisé par diverses molécules

\footnotetext{
* Dans le cas des traitements thermiques de pasteurisation et de stérilisation les manifestations de la réaction de Maillard deviennent d'autant moins accentuées que les températures utilisées sont plus élevées. En effet, la sensibilité thermique des micro-organismes devient beaucoup plus grande que celles des molécules chimiques au fur et à mesure que s'élève la température, pour l'intervalle compris entre $100^{\circ} \mathrm{C}$ et $150^{\circ} \mathrm{C}$ (Burton, 1965 ; Pien, 1972).
} 
issues de la réaction de Maillard comme le 5-hydroxy-méthyl-furfural, le méthylglyoxal ou l'acétaldéhyde (Patton, 1955) et d'une manière générale par tout ce qui va diriger la réaction de Maillard vers la dégradation de Strecker, cette étape étant connue pour son action très positive sur le brunissement (Adrian, 1973 b).

Une conservation du lait évaporé sous vide renforce également les phénomènes de coloration (Patton, 1952).

Le $\mathrm{pH}$ joue un rôle plus subtil : dans les laits tout venants le brunissement est accru par une acidification (Patton, 1955) tandis que dans les modèles expérimentaux la coloration se développe parallèlement à une augmentation du $\mathrm{pH}$ (Patton, 1952). Ce dernier point est conforme au déroulement classique de la réaction de Maillard.

L'exception apparente que constituent les laits commerciaux a été observée avec d'autres productions alimentaires comme les levures (Adrian et Frangne, 1974). On peut supposer que dans le lait - comme dans les levures - l'acidification libère des acides aminés. Leur présence renforcera alors l'intensité de la réaction de Maillard et la coloration qui en découle. Néanmoins, un autolysat enzymatique de la caséine ou du lait n'est pas responsable d'un brunissement significativement plus intense selon les observations de Patton (1952).

Enfin, la coloration du lait est indiscutablement une des manifestations de la réaction de Maillard, son développement étant en relation étroite avec la disparition de l'azote aminé libre (Richards, 1963), avec le dégagement de gaz carbonique, avec la formation de molécules réductrices et de substances fluorescentes et avec la diminution de la solubilité des poudres de lait (Tarassuk et al., 1948, 1950).

b) Autres phénomènes: A côté des molécules colorées, la réaction de Maillard est génératrice de substances fluorescentes, décelables également dans les laits traités thermiquement.

Néanmoins, dans ce milieu, la fluorescence a des origines variées : pour une part elle provient de la dégradation de la riboflavine, pour une autre elle résulte des dérivés des matières grasses et, enfin, la réaction de Maillard participe à l'apparition des substances fluorescentes (Jenness et Coulter, 1948).

En pratique, la fluorescence est étroitement associée au brunissement du lait et elle est décelable aussi bien à la suite d'une stérilisation qu'après une conservation prolongée. Dans ce domaine comme dans celui du brunissement la technique U.H.T. diminue très significativement la formation des molécules fluorescentes.

Selon Tarassuk et Simomson (1950), les protéines lactiques sont fortement impliquées dans les phénomènes de la fluorescence, ce 
qui revient à dire que la réaction de Maillard a une part importante dans cette question.

Le traitement thermique des laits liquides et la conservation des poudres sont responsables d'une acidification marquée se faisant aux dépens du lactose (Gould, 1945 ; Patton, 1950, 1952 ; Schormueller et Andraess, 1962 ; etc.). Après $8 \mathrm{~h}$ à $100^{\circ} \mathrm{C}$ un lait entier a perdu 20 p. 100 de son lactose et a gagné une acidité correspondant à $75 \mathrm{mg}$ d'acide lactique pour $100 \mathrm{ml}$. En réalité, on ne détecte que $3,5 \mathrm{mg}$ d'acide lactique formé, le reste de l'acidité étant composée de molécules engendrées classiquement par la réaction de Maillard (Gould, 1945).

C'est la raison pour laquelle, l'acidification est d'autant plus forte que les modalités expérimentales se prêtent mieux à une réaction de Maillard. Les résultats de Schormueller et Andraess (1962) montrent qu'en atmosphère humide - favorable à la réaction de Maillard - l'acidification d'une poudre de lait est nettement plus accentuée :

Valeur du pH d'une poudre de lait en fonction des modalités de chauffage

\begin{tabular}{c|c|c|c}
\hline \multirow{2}{*}{$\begin{array}{c}\text { Modalités } \\
\text { du traitement }\end{array}$} & \multicolumn{2}{|c}{ Durée du traitement (en h) } \\
\cline { 2 - 3 } & 23 & 52 & 102 \\
\hline $105^{\circ} \mathrm{C}$, en atmosphère sèche & 6,9 & 6,7 & 6,55 \\
$100^{\circ} \mathrm{C}$ en atmosphère saturée d'eau & 6,0 & 5,65 & 5,5 \\
\end{tabular}

La réaction de Maillard est classiquement responsable de l'apparition d'un pouvoir réducteur apparaissant dans les premières phases puis disparaissant par la suite.

Ces molécules réductrices proviennent de la réaction lactosecaséine ou simplement de la dégradation thermique du lactose seul (Crowe, 1948 ; Lea, 1947), c'est dire que le phénomène n'est pas spécifique de la réaction de Maillard.

L'augmentation des substances réductrices du lait a été observée à maintes reprises (Tarassuk et Jack, 1948 ; Patton, 1955), toujours en relation étroite avec les autres manifestations de la réaction de Maillard. C'est pourquoi, on peut attribuer à cette réaction la majeure 
responsabilité dans la formation des molécules réductrices décelées après chauffage ou conservation des laits.

\section{Molécules décelées à la suite des chauffages et de la réaction de Maillard}

D'une manière générale, dans un milieu complexe les traitements thermiques donnent naissance à de très nombreuses molécules que les techniques chromatographiques commencent à isoler et à inventorier (Day, 1965). En présentant les faits schématiquement, on peut classer les produits formés en trois grands groupes en fonction de leur origine. Pour s'en tenir au lait, on distingue les catégories suivantes :

a) Certaines molécules - comme les alcools furfurylique et acétique et les acides acétique et formique - semblent dériver directement de la dégradation du lactose et il n'est pas prouvé que la présence d'azote aminé soit indispensable à l'opération ni même en modifie l'intensité si l'on en juge d'après les travaux de Patton (1950, $1950 \mathrm{~b}, 1950 \mathrm{~d}$ ). Ces produits peuvent donc être considérés comme étrangers à la réaction de Maillard.

b) D'autres substances sont également décelables à la suite de la thermolyse d'un glucide ou d'un sucre pur. A titre d'exemple, la dégradation du lactose donne naissance à de l'hydroxy-méthyl-furfural (H.M.F.) par chauffage en milieu acide. Cependant, la présence d'azote aminé augmentant fortement la quantité de H.M.F. formée, cette molécule relève, partiellement de la réaction de Maillard (Patton, 1950 b, 1952). Ce type de substance peut être qualifié de "mixte » en ce sens qu'il existe en dehors de la réaction de Maillard mais que celle-ci intervient dans l'intensité de la synthèse*.

c) Certaines molécules, enfin, sont la conséquence essentielle, sinon spécifique, de la réaction de Maillard, c'est-à-dire que la présence d'acides aminés est une condition indispensable à leur apparition. Il semble que ce soit le cas, par exemple pour le maltol qui résulte d'une réaction entre le lactose et les protéines lactiques (Patton, $1952 \mathrm{c}$ ). Surtout, la dégradation de Strecker engendre des aldéhydes spécifiques provenant de la décarboxylation et de la désamination des acides aminés sous l'effet de molécules dicétoniques.

L'exemple ci-dessous, établi à partir d'observations de Jackson et Morgan (1954), fournit une illustration des mécanismes de la dégradation de Strecker telle qu'elle se déroule dans le lait, avec forma-

\footnotetext{
* Les furfurals et leurs dérivés (5-HMF) constituent les premières manifestations biochimiques d'un traitement thermique. Dans le lait - comme dans de très nombreuses matières alimentaires - leur présence est l'indice précurseur d'une réaction de Maillard, avec les conséquences qu'elle entraîne dans le domaine protidique (Keeney et Bassette, 1959 ; Craig et al., 1961 ; ete.).
} 
tion d'aldéhydes aromatiques en $\mathrm{Cn}-1$ par rapport aux acides aminés mis en jeu :

$$
\begin{aligned}
& \begin{array}{c}
\mathrm{R}-\mathrm{C}-\mathrm{C}-\mathrm{R}: \\
\text { II } \\
\mathrm{O} \text { O } \\
\text { Réductones }
\end{array} \\
& \text { (ac. ascorbique, } \\
& \text { ac. pyruvique, } \\
& \text { glyoxal, etc.) } \\
& \mathrm{R}-\underset{\text { II }}{\mathrm{C}}-\underset{\mathrm{O}}{\mathrm{C}} \stackrel{\mathrm{NH}}{\mathrm{C}}-\mathrm{R}_{2} \\
& \begin{array}{l}
+\mathrm{CH}_{3}-\underset{l}{\mathrm{CH}}-\mathrm{CH}_{2}-\underset{\mathrm{CH}_{3}}{\mathrm{CH}}-\mathrm{CO}_{2} \mathrm{H} \\
\begin{array}{c}
\text { Acide aminé } \\
\text { (leucine) }
\end{array} \\
+\mathrm{CH}_{3}-\underset{\mathrm{CH}}{\mathrm{CH}}-\mathrm{CH}_{2}-\mathrm{CHO}+\mathrm{CO}_{2} \\
\mathrm{CH}_{3}
\end{array} \\
& \text { Aldéhyde } \\
& \text { (méthylbutanal) }
\end{aligned}
$$

D'autres molécules sont également la marque indéniable de la réaction de Maillard : on peut citer les 1-amino 1-déoxy 2-cétoses étudiées dans les poudres de lait et dans les systèmes lactose-caséine par Richards (1963). De même, ont été précisé les molécules résultant de la combinaison de la lysine avec le lactose ou le lactulose (Erbersdobler et al., 1966, 1967 ; Finot et al., 1968, 1969 ; Erbersdobler, 1970) : lorsqu'elle est soumise à une hydrolyse acide classique, la lysine bloquée par un sucre comme le lactose donne naissance à deux dérivés, le furosine et la pyridosine (fig. 3). Le premier d'entre eux,

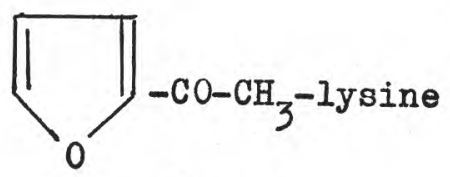

\section{Furosine}
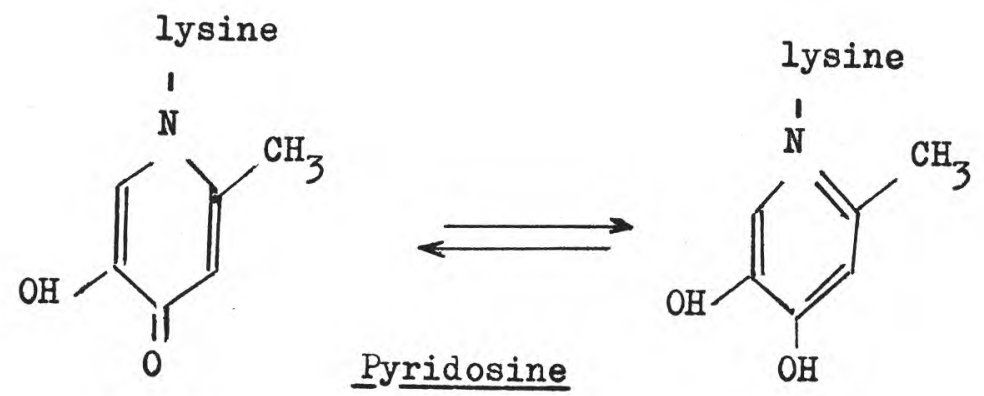
particulièrement abondant, peut représenter jusqu'à la moitié de la lysine présente dans une poudre de lait. Son taux est lié à celui de la lysine disponible par la relation suivante (Erbersdobler 1970) :

$$
\text { furosine }=3,16-0,42 \text { (lysine disponible) }
$$

Tels sont les principaux types de molécules susceptibles d'être détectées à la suite d'une réaction de Maillard se déroulant dans un produit laitier. En dresser une liste exhaustive représentait une tâche utopique jusqu'à une époque récente et seules les techniques modernes de chromatographie permettent d'aborder ce problème. Ferretti et Flanagan $(1970,1971,1972)$ ont procédé à des analyses de la fraction volatile de mélange lactose-caséine, de poudre de lactosérum ou de poudre de lait écrémé ayant subi des réactions de Maillard dans des conditions diverses. Dans la poudre de lactosérum, 26 substances attribuables, en majorité, à la réaction de Maillard ont pu être identifiées, 40 dans le système expérimental lactosecaséine et 43 dans une poudre de lait après plus de 2 ans de conservation. Dans le mélange caséine-lactose ont été isolés 13 composés furaniques, 9 lactones, 5 pyrazines, 2 pyridines, 2 amides, 2 acides carboxyliques, 2 dérivés pyrroliques, de l'acétone, du maltol, etc. D’un autre côté, la réaction de Maillard se déroulait grâce à la présence de galactose, de tagatose, de lactulose en dehors de l'action propre du lactose initial.

Sur le plan quantitatif, les composés les plus abondants sont :

- dans le système lactose-caséine : les acide et alcool acétiques, le 2-acétylfurane, le 5-méthyl 2-furaldéhyde, les acétate et alcool furfuryliques, la butyrolactone, l'hydroxybutyrolactone et le maltol,

- dans la poudre de lait écrémé : les acides butyrique, proprionique et hexanoïque, la diméthylsulfone, puis à une concentration moindre, des diméthylpyrazines, du maltol, de la furaldéhyde et de la benzaldéhyde, des acides benzoïque, isobutyrique et acétique, des dérivés benzéniques, des alcanes, des lactones, etc.

A côté de ces molécules de nature très diverse et dont l'origine précise demeure difficile à établir, on doit mentionner l'apparition de substances apportant une preuve indéniable de la réaction de Maillard, il s'agit des aldéhydes provenant des acides aminés ayant réagi dans la dégradation de Strecker et qui sont des molécules spécifiques de la réaction de Maillard.

Ces aldéhydes ont été identifiés dans des poudres de lait (Ramshaw et Dunstone, 1969) ou bien dans une solution de caséine autoclavée $1 \mathrm{~h}$ à $140^{\circ} \mathrm{C}$, fournissant la démonstration d'une possibilité de réaction entre la partie azotée de la caséine et sa fraction glucidique (Nakanishi et Itoh, 1967) : 


\begin{tabular}{l|l}
\hline \multicolumn{1}{c|}{ Aldéhyde identifié } & \multicolumn{1}{|c}{ Origine probable } \\
\cline { 2 - 2 } & \\
Méthanal & Glycocolle \\
Ethanal & Alanine \\
Propanal & Thréonine (?) \\
Butanal ou crotonal & Acide glutamique (?) \\
Méthional & Méthionine \\
2-méthyl propanal & Valine \\
3-méthyl butanal & Leucine \\
2-méthyl butanal & Isoleucine \\
Phénylacétaldéhyde & Phénylalanine \\
& \\
\hline
\end{tabular}

En bref, la réaction de Maillard est indiscutablement responsable de l'apparition de nombreuses molécules dans les produits laitiers chauffés ou conservés. Néanmoins, elle ne représente pas le seul mécanisme en cause ; elle se développe conjointement avec d'autres processus comme la dégradation du lactose ou l'oxydation des lipides résiduels. Ces divers mécanismes peuvent - de surcroît interférer entre eux : à titre d'illustration, les aldéhydes des acides gras (produit d'oxydation) réagissent avec des acides aminés et sont, en conséquence, un facteur de la réaction de Maillard.

\section{RESPONSABILITE DE LA REACTION DE MAILLARD DANS LA FORMATION DES AROMES}

Dans la mesure où il est possible de schématiser des questions aussi subtiles que celle des arımes et des flaveurs, on peut avancer qu'il existe deux grands types de flaveurs dans les produits laitiers traités industriellement. Le premier et le plus fréquent est un goût de cuit, de vieux, d'oxydé (sunlight flavor). Il ne relève pas de la réaction de Maillard mais essentiellement des processus de destruction de la riboflavine, de dénaturation des protéines solubles et surtout de l'évolution des groupements sulfhydriles volatils (Zweig et Block, 1953 ; Kinsella, 1971 ; etc.).

La seconde flaveur décelable est la conséquence de la dégradation du lactose et de la réaction de Maillard qui y est associée. On la décèle surtout à la suite de la conservation de poudres de lait ou lors de traitements thermiques sévères (Tarassuk et Jack, 1948 ; Coulter et al., 1952 ; Ramshaw et Dunstone, 1969 ; etc.).

Quelques relations ont été établies entre l'évolution physicochimique des produits laitiers et leur flaveur. Les observations de Tarassuk et Jack (1948) ont notamment le mérite de faire apparaître 
une distinction entre la flaveur développée à la suite d'une évolution banale de la poudre de lait et celle qui correspond plus précisément à l'existence d'une réaction de Maillard :

\begin{tabular}{l|c|c|c}
\hline \multirow{2}{*}{ Goût } & $\begin{array}{c}\text { Indice de } \\
\text { brunissement }\end{array}$ & Pression (en mm) de \\
\cline { 3 - 3 } & 0,8 & 3,5 & oxygène \\
\cline { 3 - 3 } Bon & 1,5 & 19,0 & 61,0 \\
Vieux & 11,5 & 199,0 & 1,0 \\
Caramel & & 1,0 \\
\hline
\end{tabular}

Les conditions d'apparition d'un goût particulier de céréale ont été analysées à plusieurs reprises. Cette flaveur se décèle à la suite d'un chauffage qui a produit la dénaturation de la moitié des protéines solubles (Kinsella, 1971) et l'apparition d'hydroxy-méthyl-furfural à des concentrations de l'ordre de 10 à 15 micromoles par litre de lait (Keeney et Bassette, 1959 ; Craig et al., 1961).

Les autres molécules volatiles engendrées par la dégradation du lactose comme par la réaction de Maillard participent au développement de la flaveur des produits laitiers chauffés. L'importance de leur rôle dépend à la fois de leur concentration dans le produit et de leur seuil de perception organoleptique.

En fonction de ce double critère, les composés qui ont le plus de probabilités d'intervenir dans l'élaboration de la flaveur sont les suivants, d'après Ferretti et Flanagan (1972) : 2-furaldéhyde, 2-furfuryl butyrate, méthyl éthyl pyrazine, triméthyl pyrazine, N-éthyl 2-formylpyrrole, crésol, benzaldéhyde, salicylaldéhyde, cumène, naphthalène, benzothiazole et 2-nonanone.

Enfin, des évolutions notables de flaveur peuvent être observées dans des caséines conservées dans des conditions favorables à la réaction de Maillard (10 p. 100 d'humidité) : après 3 mois, une altération importante est observée avec un taux de lactose résiduel de 1 p. 100 se traduisant par une forte apparition de H.M.F. et de molécules réductrices et par une chute de la qualité organoleptique (tab. 7).

La présence des glucides constitutifs de la caséine semble même suffire pour déclencher une réaction de Maillard et en réduire la valeur organoleptique, la caséine-présure présentant une stabilité 


\section{TABLEAU 7}

Evolution de la caséine au cours d'une conservation de 3 mois, en fonction de son taux de lactose résiduel*

\begin{tabular}{c|c|c|c}
\hline $\begin{array}{c}\text { Taux de lactose } \\
\text { (p. 100) }\end{array}$ & $\begin{array}{c}\text { H-M-F } \\
\text { (a) }\end{array}$ & $\begin{array}{c}\text { Substances } \\
\text { réductrices } \\
\text { (b) }\end{array}$ & $\begin{array}{c}\text { Qualité } \\
\text { organoleptique } \\
\text { (c) }\end{array}$ \\
\hline 0,01 & 0,021 & 0,043 & 5,1 \\
0,20 & 0,023 & 0,032 & 5,2 \\
1,00 & 0,176 & 0,363 & 1,6 \\
\hline
\end{tabular}

N.B. - Le produit renferme de l'ordre de 10 p. 100 d'eau et présente une qualité organoleptique de 7 environ au moment de la fabrication.

(a) Estimé en acide thiobarbiturique.

(b) Estimées en ferricyanure.

(c) Echelle de 10 (excellent) à 1 (très mauvais).

* d'après Walker (1972).

franchement supérieure à celle des autres formes, même à taux de lactose résiduel identique (Walker, 1972).

\section{CONCLUSIONS}

L'application de traitements thermiques aux produits laitiers se traduit par des modifications physico-chimiques des fractions protidiques et par des réactions chimiques dans lesquelles entrent les protéines solubles et la caséine.

- Au point de vue physico-chimique, les traitements thermiques les plus modérés sont seulement responsables d'une dénaturation des protéines solubles du lactosérum. Les chauffages intenses peuvent modifier à la fois la structure et la composition des caséines : les particules tendent à augmenter de taille et à s'insolubiliser sous forme de caséinate de calcium ; en même temps on assiste à une déminéralisation de la fraction de caséine non précipitée (perte de phosphore et de calcium).

- Au point de vue chimique, les produits laitiers offrent une sensibilité extrême à la réaction de Maillard en raison de la réactivité du lactose et des protéines du sérum. 
Les phénomènes sont particulièrement intenses dans les milieux peu hydratés et la réactivité maximale s'observe dans les poudres de lait contenant plus de 5 p. 100 d'eau. Les laits liquides présentent infiniment moins de risques étant donné leur forte hydratation, facteur inhibiteur de la réaction de Maillard.

Celle-ci se produit lors des traitements thermiques mais également au cours d'une conservation prolongée.

La réaction de Maillard provoque la destruction sélective des acides aminés basiques et fait apparaître assez rapidement la lysine comme facteur limitant primaire. Elle engendre de nombreuses molécules modifiant les caractères physico-chimiques des produits laitiers : coloration, fluorescence, acidification et pouvoir réducteur.

La réaction de Maillard, et la dégradation de Strecker, donnent naissance à des substances plus ou moins spécifiques qui participent à la formation d'un goût de " caramel " décelable dans les produits lactiques chauffés ou conservés. Cette flaveur est différente du goût de " vieux ", de " cuit " ou d' " oxydé " décelables également dans les laits mais qui relèvent d'autres mécanismes.

\section{S u m m a r y}

The application of the thermic treatments to the dairy products is expressed in physico-chemical modifications of protein fractions and in chemical reactions including the soluble proteins and the casein.

- From a physico-chemical point of view, the most moderate thermic treatments are only accountable for a denaturation of soluble protein of the whey. Intense heatings may modify both the structure and composition of caseins ; these particles are inclined to increase and to render themselves insoluble in the form of caseinates; at the same time, there is an unmineralization of the undenatured fraction of casein (loss in phosphorus and calcium).

- From a chemical point of view, the dairy products present an extreme sensibility to the Maillard reaction because of the lactose and the whey proteins reactivities.

Phenomena are particularly intense in the media which are not very hydrated and the maximum reactivity can be seen in milk powder containing more than $5 \mathrm{p} .100$ of water. Liquid milks offer fewer risks by reason of their important hydration, inhibitory factor of the Maillard reaction.

This latter takes place during the thermic treatments but also during a prolonged conservation. 
The Maillard reaction provokes the selective destruction of the basic amino-acids and makes lysin comparatively rapidly, as a primary limiting factor.

It develops numerous molecules modifying the physico-chemical characters of the dairy products : coloration, fluorescence, acidification and reducing power.

The Maillard reaction and the Strecker degradation provoke more or less specific substances which have a share in the formation of burnt-sugar taste and are likely to be found in heated or preserved dairy products. This flavor is different from other tastes of " old ", " cooked ", " sun flavor ", which are dependent on other mechanisms.

\section{Bibliographie}

AdRIAN (J.) (1967). - Ann. nutrit. alim., 21, 129-47.

Adrian (J.) (1972). - Ind. alim. agric., 89, 1281-9 et 1713-20 ; idem, 1973, 90, 449-55 et 559-64; Wordl rev. nutrit, diet., 1974, 19, 71-122.

Adrian (J.) (1973). - La valeur alimentaire. Un vol., Paris, La Maison Rustique, édit.

AdRIAN (J.) (1973 b). - Ann, nutrit. alim., 27, 299-314.

AdRian (J.), Frangne (R). (1969), - Ind. alim. agric., 86, 801-6.

AdRiAn (J.), Frangne (R.) (1975). - Ind. alim. agric. (sous presse).

Adrian (J.), Petit (L.) (1970). - Ann. nutrit. alim., 24, B131-B168.

Alais (C.), Jollès (P.), Kiger (N.) (1966). - 17e Congr. intern. lait., B 2, 341-8.

Alais (C.), Kiger (N.), Jollès (P.) (1967). - J. dairy sci., 50, 1738-43.

Belec (J.), Jenness (R.) (1962). - J. dairy sci., 45, 12-9; 20-6.

BJARnason (J.), CARPENTER (K. J.) (1970). - Brit. J. nutrit., 24, 313-29.

Block (R. J.), JoNes (D. D.), GERSDORFF (C.E.F.) (1934). - J. biol. chem., 105, 667-8.

BoCK (H. D.), WuENSCHE (J.) (1965). - Nahrung, 9, 131-5.

Van den Bruel (A.M.R.), Jenneskens (P. L.), Mol (J. J.) (1972). - Netherl. milk dairy J., 26, 19-30.

Brueggemann (J.), ERbersdobler (H.) (1968). - Z. Tierphysiol. Tierernaehr. Futtermittelk, 24, 55-67, 136-52.

Burton (H.) (1965). - J. soc. dairy technol., 18, 58-65.

Causeret (J.), Lhuissier (M.), Hugot (D.) (1970). - Ann. nutrit. alim., 24, B169B200.

Causeret (J.), Mocouot (G.) (1964). - Ann. nutrit. alim., 18, C267-C354.

Champagnat (A.), Adrian (J.) (1974). - Pétrole et protéines. Un vol., Paris, Doin, édit.

Chatelus (C.) (1964). - Bull. chim. France, 2523-32 ; idem., 1965, 929-33.

Cheeke (P. R.), StAngel (D. E.) (1972), - J. anim. sci., 34, 757-61.

Chick (Dame-) (H.), Boas-Fixsen (N. A.), Hutchinson (J.C.D.), Jackson (H. M.) (1935). - Biochem. J., 29, 1712.

Cook (B. B.), Morgan (A. F.), Singer (B.), Parker (J.) (1951). - J. nutrit., 44, 63-81. 
CoOK (B. B.), Fraenkel-Conrat (J.), Singer (B.), Morgan (A. F.) (1951 b). - J. nutrit. $44,217-35$.

Cook (B. B.), Morgan (A. F.), Weast (E. O.), Parker (J.) (1951 c). - J. nutrit., 44, 51-61.

Coulter (S. T.), Jenness (R.), Geddes (W. F.) (1952), - Adv. food res., 3, 45-118.

Craig (J. C.) jr, Aceto (N. C.), Della Monica (E. S.) (1961). - J. dairy sci., 44, 1827-35.

CRowe (L. K.), Jenness (R.), Coulter (S. T.) (1948), - J. dairy sci., 31, 595-610.

Damicz (W.), Budlawski (J.), Pogorzelski (K.) (1965). - Le Lait, nº 447, 379-86.

DAY (E. A.) (1965). - Food technol., 19, 1585-90.

Mc Donald (F. J.) (1966). - Nature, 209, 1134.

Dulcino (J.), Lontie (R.) (1959). - 7e Coll. prot. biol. fluids, Bruges, 100-4.

Dupont (M.) (1965). - Biochem. biophys. Acta, 94, 573-5.

EHLE (S. R.), JANSEN (G. R.) (1965). - Food technol., 19, 1435-9 ; 1439-42.

ERBERSDOBLER (H.) (1970), - Milchwissenschaft, 25, 280-4.

ERbersdobler (H.), BocK (G.) (1967). - Naturwissenschaft, 54, 648.

Erbersdobler (H.), ZucKer (H.) (1966). - Monat. Futterm. Wirtschaft, 49, $\mathrm{n}^{\circ} 7$; idem, Milchwissenschaft, 21, 564-8.

Evans (R. J.), Bandemer (S. L.), Bauer (D. H.) (1961). - J. food sci., 26, 663-9.

Ferretti (A.), Flanagan (V. P.), Ruth (1970). - J. agric. food chem., 18, 13-8.

Ferretti (A.), Flanagan (V.P.) (1971). - J. dairy sci., 54, 1769-71; idem, J. agric. food chem., 19, 245-9.

Ferretti (A.), Flanagan (V. P.) (1972). - J. agric. food chem., 20, 695-8.

FINK (H.) (1959). - Milchwissenschaft, 14, 323-5 et 325-7.

FINK (H.) Schlie (I.) (1963). - Nahrung, 7, 277-99.

FINK (H.), SCHLIE (I.), RUGE (U.) (1958). - Naturforsch., 13 B, 610-6.

Finot (P.A.), Viani (R.), Bricout (J.), Mauron (J.) (1968). - Experientia, 24, 1097-9.

Finot (P. A.), Viani (R.), Bricout (J.), Mauron (J.) (1969). - Experientia, 25, 134-5.

Ford (J. E.), Porter (J. W. G.), BURTON (H.) (1966). - 17e Congr. intern. lait., B 2, 357-60.

Fox (K. K.), Harper (M. K.), Holsinger (V. H.), Pallansch (M. J.) (1967). - $J$. dairy sci., 50, 443-50.

Fox, (K. K.), Holsinger (V. H.), Posati (L. P.), Pallansch (M. J.) (1967 b). J. dairy sci., 50, $1032-7$.

Frangne (R.) (1972). - Diplôme EPHE, Paris.

FrangNe (R.), AdRian (J.) (1967). - Ann. nutri. alim., 21, 163-74.

Frangne (R.), Adrian (J.) (1972). - Ann. nutrit, alim., 26, 97-106.

Gouch (P.), JENNESS (R.) (1962), - J. dairy sci., 45, 1033-9.

Gould (I. A.) (1945). - J. dairy sci., 28, 367-77.

Greaves (E. O.), Morgan (A. F.) (1933), - Proc. soc. exp. biol. med., 31, 506-7.

Gregory (M. E.), Henry (K. M.) Kon (S. K.) (1964). - J. dairy res., 31, 113-9.

Guy (E. J.), Vettel (H. E.), Pallansch (M. J.) (1967). - J. dairy sci., 50, 828-32.

Harland (H. A.), Coulter (S. T.), Jenness (R.) (1952). - J. dairy sci., 35, 363-8.

Henry (K. M.), Houston (J.) ,Kon (S. K.), Thompson (S. Y.) (1944). - J. dairy res., 13, 329-39. 
Henry (K. M.), Kon (S. K.), LeA (C. H.), Smith (J. A. B.), White (J. C. D.) (1946). Nature, $158,348$.

Henry (K. M.), Kon (S. K.), Rowland (S. J.) (1946 b). - J. dairy res., 14, 403-14,

HENRY (K. M.), KoN (S. K.) (1947). - J. dairy res., 15, 140-1.

Henry (K. M.), Kon (S. K.), LeA (C. H.), White (J. C. D.) (1948), - J. dairy res., 15, 292-363.

Hindle (E. J.), Wheelock (J. V.) (1970). - J. dairy res., 37, 397-405.

Holm (H.) (1971). - J. sci. food agric., 22, 378-81.

Hostettler (H.), LANg (K.), CzoK (G.), Fricker (A.), Griem (W.), IMHoF (K.), Kiekebusch (W.), Krug (E.), PABSt (W.), SteIN (J.) (1965). - Z. Ernaehrungswissenschaft, 6, 25-35.

Hostettler (H.), IMHof (K.), Stein (J.) (1965 b). - Milchwissenschaft, 20, 189-98.

Howat (G. R.), Wright (N. C.) (1934). - Biochem. J., 28, 1336-45.

Hugot (D.), CAuseret (J.) (1962). - Ann. technol. agric., 11, 55-62.

Jackson (H. W.), MoRgan (M. E.) (1954). - J. dairy sci., 37, 1316-24.

Jenness (R.), Coulter (S. T.) (1948), - J. dairy sci., 31, 367-81.

KARTE (H.) (1966). - Milchwissenschaft, 11, 420-7.

KeEney (M.), BASSETTE (R.) (1959). - J. dairy sci., 42, 945-60.

KieRCHMEIER (O.) (1962). - Milchwissenschaft, 17, 408-12.

KisZA (J.), Sobina (A.), Zвiкоwsкi (Z) (1966). - 17e Congr. intern. lait., E 3, 85-9.

KRAFT (R. A.), MoRgan (A. F.) (1952). - J. nutrit., 45, 567-81.

Lang (K.), CZoK (G.), Fricker (A.), Griem (W.), Kiekebusch (W.), Krug (E.), PABST (W.) (1965). - Milchwissenschaft, 20, 309-15.

LEA (C. H.) (1947). - Analyst, 72, 336-9.

Loncin (M.), Jacomain (D.), Tutindjian-Pruvost (A. M.), Lenges (J. P.), Bimbenet (J.) (1965). - C. R. Acad. Sci., 260, 3208-11.

Lontie (R.), Préaux (G.) (1966). - 36 Congr. intern. chim. industr., Bruxelles, 32 III.

Mader (I. J.), Schroeder (L. J.), Smith (A. H.) (1949). - J. nutrit., 39, 341-55.

Mauron (J.), Mottu (F.), Bujard (E.), Egli (R. H.) (1955). - Arch. biochem. biophys., 59, 433-51.

Mélachouris (N. P.), Tuckey (S. L.) (1966). - J. dairy sci., 49, 1154-6.

MORR (C. V.) (1965). - J. dairy sci., 48, 29-33.

MORR (C. V.) (1969). - J. dairy sci., 52, 1174-80.

MORR (C. V.), JosephSON (R. V.) (1968). - J. dairy sci., 51, 1349-55.

Murthy (L.), Herreid (E. O.), Whitney (R. Mc L.) (1958). - J. dairy sci., 41, 1324-41.

NAKANishi (T.), ІтоH (T.) (1966). - Milchwissenschaft, 21, 635-7.

NaKanishi (T.), IтоH (T.) (1967). - J. agric. chem. soc. Jap., 41, 130-4.

Nakanishi (T.), IтоH (T.) (1967 b). - Agric. biol. chem., 31, 1066-9.

Patton (S.) (1950.) - J. dairy sci., 33, 324-8.

Patton (S.) (1950 b). - J. dairy sci., 33, 904-10.

Patton (S.) (1950 c). - J. dairy sci., 33, 102-6.

Patton (S.) (1950 d). - J. dairy sci., 33, 526-30.

Patton (S.) (1952). - J. dairy sci., 35, 1053-66.

Patton (S.) (1955). - I. dairy sci., 38, 457-78. 
Payens (T. A. J.) (1966). - J. dairy sci., 49, 1317-24.

Payne-Botha (S.), Bigwood (E. J.) (1959). - Brit. J. nutrit., 13, 385-9.

Pien (J.) (1972). - Bull. F.I.L., Bruxelles.

Pion (R.), Rerat (A.) (1962). - 16 ${ }^{\mathrm{e}}$ Congr. intern. lait., $V$ 2, 993-1001.

PongPaew (P.), GugGenheim (K.) (1968). - Nutrit. diet., 10, 297-308.

PORTER (J. W. G.) (1966). - J. soc. dairy technol., 19, 1-7.

Prahl (L.), TAuefel (K.) (1967). - Nahrung, 11, 257-65.

Ramshey (E. H.), Dunstone (E. A.) (1969). - J. dairy res., 36, 215-23.

RAmShey (E. H.), Dunstone (E. A.) (1969 b). - J. dairy res., 36, 203-14.

Rao (M. N.), Sreenivas (H.), Swaminathan (M.), Carpenter (K. J.), Morgan (C. B.) (1967). - J. assos. off. anal. chem., 50, 704-7.

RichaRDS (E. L.) (1963). - J. dairy res., 30, 223-34.

Rose (D.) (1962). - J. dairy sci., 45, 1305-11.

Ross (I.), Krampitz (G.) (1960). - Z. Tierphysiol. Tierernaehr. Futtermittelk., 15, 95-101.

SAWyer (W. H.), Coulter (S. T.), Jenness (R.) (1963). - J. dairy sci., 46, 564-5.

SCHILleR (K.) (1956). - Z. Tierphysiol. Tierernaehr. Futtermittelk., 11, 264-7.

Schmidt (D. G.) (1969). - Netherl. milk dairy J., 23, 128-35.

Schormueller (J.), ANdraess (W.) (1962). - Z. Lebensmittel Unters. Forsch., 118, $12-22$.

Schroeder (L. J.), Stewart (R. A.), Smith (A. H.) (1951). - J. nutrit., 45, 61-74.

Shillam (K. W. G.), DaWson (D. A.), Roy (J. H. B.) (1960). - Brit. J. nutrit., 14, 403-12.

Shillam (K. W. G.), Roy (J. H. B.), Ingram (P. L.) (1962). - Brit. J. nutrit., 16, 267-77.

Shillam (K. W. G.), Roy (J. H. B.) (1963). - Brit. J. nutrit., 17, 171-81.

Swirski (M.), Allouf (R.), Cheftel (H.) (1962). - Le Lait, 42, 260-7 ; 392-405.

TAGaRI (H.), Roy (J. H. B.) (1969). - Brit. J. nutrit., 23, 763-82.

TARASSUK (N. P.), JACK (E. L.) (1948). - J. dairy sci., 31, $255-68$.

TARASSUK (N. P.), Simonson (H. D.) (1950). - Food technol., 4, 88-92.

Tsugo (T.), Yamauchi (K.), Yoshino (U.) (1961). - Nippon Nageikagaku kaishi, $35,888$.

De Vuyst (A.), Vervack (W.), Charlier (H.), Jadin (V.) (1971), - Bull. acad. roy. méd. belge, 11, 231-46.

WALKER (N. J.) (1972). — J. dairy res., 39, 231-8.

ZaDow (J. G.) (1969). - Austral J. dairy technol., 24, 449.

ZitTle (C. A.) (1969). - J. dairy sci., 52, 1356-8.

ZWeIG (G.), Block (R. J.) (1953). - J. dairy sci., 36, 427-36. 